\title{
Manejo perioperatorio de la anticoagulación en cirugía cardíaca: ¿un lugar para los anticoagulantes directos?
}

\author{
Dr. Pablo Asadurian Tchakirian
}

\section{Resumen}

La anticoagulación en el posoperatorio de cirugía cardíaca constituye todo un reto. La oportunidad de inicio, la dosis a utilizar y el tipo de anticoagulante, dependerán del procedimiento realizado, de la asociación de factores de riesgo trombótico y/o hemorrágico y de un estricto seguimiento clínico, a fin de lograr un adecuado nivel de protección y evitar complicaciones durante la estadía hospitalaria. Analizaremos tres escenarios de posoperatorio: pacientes con prótesis valvulares, fibrilación auricular y prevención de la enfermedad tromboembólica venosa, así como la prevención y el manejo de las complicaciones hemorrágicas, adecuando el tratamiento a los niveles de INR y a la presencia de sangrados. El trabajo en equipo, multi e interdisciplinario y la protocolización de las medidas a tomar, facilitan el manejo de este tipo de pacientes y reducen las complicaciones, muchas de ellas totalmente evitables.

Palabras clave: ANTICOAGULACIÓN POSOPERATORIO DE CIRUGÍA CARDÍACA

WARFARINA

ANTICOAGULANTES DIRECTOS

\section{Perioperative management of anticoagulation in cardiac surgery: a place for} direct anticoagulants?

\section{Summary}

Anticoagulation in the postoperative period of cardiac surgery constitutes a challenge. The timing of initiation, the anticoagulation dose to be used and the type of anticoagulant will depend on the procedure performed, the association of thrombotic and/or hemorrhagic risk factors, and strict clinical monitoring in order to achieve an adequate level of protection and avoid complications during hospital stay. We will analyze three postoperative situations: patients with valve prostheses, atrial fibrillation and prevention of venous thromboembolic disease as well as prevention and management of bleeding complications, adapting treatment to INR levels and presence of bleeding. Multi and interdisciplinary teamwork and the protocolization of the measures to be taken, facilitate the management of these patients and reduces complications, many of which are totally avoidable.

Key words: $\quad$ ANTICOAGULATION

POSTOPERATIVE CARDIAC SURGERY

WARFARIN

DIRECT ANTICOAGULANTS

\section{Gerenciamento perioperatório de anticoagulação em cirurgia cardíaca: ¿um} lugar para anticoagulantes diretos?

\section{Resumo}

A anticoagulação no pós-operatório de cirurgia cardíaca constitui um desafio. O momento de início, a dose de anticoagulação a ser utilizada, o tipo de anticoagulante vão depender do procedimento realizado, da associação de fatores de risco trombóticos e/ou hemorrágicos e de monitoramento clínico rigoroso para atingir um nível adequado de proteção e evitar complicações durante a internação. Analisaremos três cenários pós-operatórios: pacientes com próteses valvares, fibri-

Instituto Nacional de Cirugía Cardíaca (INCC). Montevideo, Uruguay.

Correspondencia: Dr. Pablo Asadurian Tchakirian. Correo electrónico: asadurian@gmail.com

El autor declara no tener conflictos de intereses.

Recibido Oct 2, 2020; aceptado Oct 11, 2020 
lação atrial e prevenção de doença tromboembólica venosa, além da prevenção e manejo de complicações hemorrágicas, adequando o tratamento aos níveis de INR e à presença de sangramento. $\mathrm{O}$ trabalho em equipe multi e interdisciplinar $\mathrm{e}$ a protocolização das medidas a serem tomadas facilitam o manejo destes pacientes e reduzem complicações, muitas das quais são totalmente evitáveis.

$\begin{array}{ll}\text { Palavras chave: } & \text { ANTICOAGULAÇÃO } \\ & \text { PÓS-CIRURGIA CARDÍACA } \\ & \text { WARFARIN } \\ & \text { ANTICOAGULANTES DIRETOS }\end{array}$

\section{Introducción}

La prevalencia de cirugía de revascularización miocárdica (CRM) se sitúa en 17-74 casos cada 100.000 habitantes, según diferentes estadísticas, y 60 cada 100.000 habitantes reciben sustitución de válvula aórtica (SVA), aunque esta última cifra viene en franco descenso dado el advenimiento del implante percutáneo de válvula aórtica (TAVI) ${ }^{(1-2)}$.

La necesidad de utilización de anticoagulantes en el posoperatorio de cirugía cardíaca (POCC) representa todo un reto: requiere un adecuado balance riesgo-beneficio, (riesgos hemorrágico y trombótico), definir oportunidad de inicio, establecer controles clínicos y paraclínicos, anticipar complicaciones hemorrágicas y el tratamiento adecuado en caso de que ocurran. Cada paciente deberá ser tratado en forma personalizada. Una hemorragia constituye factor pronóstico adverso que determina la obligada suspensión de la terapia anticoagulante, con el riesgo potencial de trombosis que deseábamos prevenir.

Analizaremos tres escenarios posibles en el POCC:

- Prótesis valvulares.

- Fibrilación auricular (FA) posoperatoria.

- Prevención de enfermedad tromboembólica venosa.

\section{La hemostasis en el posoperatorio}

La circulación extracorpórea (CEC) afecta el sistema de la coagulación, activa los neutrófilos, linfocitos y plaquetas. Se libera complemento, aumentando los niveles de C3 cinco veces por encima de los valores del preoperatorio. Hay liberación de citoquinas, factor tisular y se produce activación del factor VII, iniciando la cascada de la coagulación. Como contrapartida, también hay activación de la fibrinólisis, que predispone al sangrado. Todo este proceso, que involucra inflamación y alteraciones del sistema de la coagulación, genera grandes desafíos al momento de preservar el equilibrio entre sangrado y trombosis. Además, la CEC determina hemodilución, anemia y plaquetopenia que en general se recupera en 48-72 horas, a diferencia de la tromboci- topenia inducida por heparina (HIT) a la que luego haremos referencia ${ }^{(3)}$

\section{Principios básicos de prescripción y control de la anticoagulación}

La dosis de anticoagulante a utilizar dependerá de la presencia de factores de riesgo para trombosis o hemorragia y del motivo de anticoagulación.

En el caso de las prótesis valvulares, se reconocen como factores de riesgo trombótico, tipo y diseño, (prótesis mecánica > que biológica), posición y número, y tiempo transcurrido desde el implante. El flujo transvalvular a nivel del anillo aórtico es mayor que a nivel mitral, donde existe mayor estasis circulatoria y, en consecuencia, mayor riesgo trombótico.

La doble prótesis mecánica mitro-aórtica aumenta el riesgo anual (0,6 \% para una prótesis vs $3,3 \%$ para 2) ${ }^{(4)}$.

La asociación de FA, disfunción del ventrículo izquierdo, aurícula izquierda dilatada, estados protrombóticos, y antecedentes de complicaciones tromboembólicas, son todos factores de riesgo adicionales que puede modificar la dosis de anticoagulante a utilizar (tabla 1).

De la misma manera debe determinarse el riesgo hemorrágico, que es promedialmente del $1 \%$ anual, siendo mayor en pacientes con prótesis mecánica mitral, dado que requiere rangos más altos de anticoagulación. También incrementan el riesgo la necesidad de terapia antiplaquetaria, mayor edad, presencia de discrasias sanguíneas, e influyen la ocupación laboral del paciente y su accesibilidad a los controles, el antecedente de enolismo e insuficiencia hepática, entre otros ${ }^{(4)}$.

Una vez identificada la necesidad de tratamiento anticoagulante, se debe definir:

- Oportunidad de inicio.

- Controles de INR en caso de warfarina.

- Nivel óptimo de anticoagulación.

- Prevención y tratamiento precoz de las complicaciones hemorrágicas.

La heparinoterapia se inicia entre las $24-48$ horas del posoperatorio, preferiblemente con heparinas de bajo peso molecular (HBPM), una vez extraí- 


\begin{tabular}{|c|c|c|c|}
\hline \multirow[t]{2}{*}{ Riesgo } & \multicolumn{3}{|c|}{ Motivo de la anticoagulación } \\
\hline & Válvulas mecánicas & Fibrilación auricular & Tromboembolia venosa \\
\hline \multirow[t]{3}{*}{ Alto } & Posición mitral & $\mathrm{CHA}_{2} \mathrm{DS}_{2}$-VASc 7-9 & TEV reciente $(<3$ meses $)$ \\
\hline & $\begin{array}{l}\text { Tricúspide (incluso } \\
\text { biológica) }\end{array}$ & $\mathrm{ACV} / \mathrm{AIT}<3$ meses & \multirow{2}{*}{$\begin{array}{l}\text { Trombofilia grave } \\
\text { (homocigosis FV Leiden, } \\
20210 \text { protrombina, déficit } \\
\text { de proteína C, S o } \\
\text { antitrombina III, defectos } \\
\text { múltiples, SAF) }\end{array}$} \\
\hline & $\begin{array}{l}\text { Aórtica (modelo antiguo) } \\
\text { ACV/AIT < } 6 \text { meses }\end{array}$ & $\begin{array}{l}\text { Valvulopatía mitral } \\
\text { reumática }\end{array}$ & \\
\hline Moderado & $\begin{array}{l}\text { Posición aórtica }+1 \text { FR } \\
\text { (FA, ACV/AIT previo }>6 \\
\text { meses, DM, IC, edad }>75 \\
\text { años) }\end{array}$ & $\begin{array}{l}\mathrm{CHA}_{2} \mathrm{DS}_{2}-\mathrm{VASc} 5-6 \\
\text { ACV/AIT > } 3 \text { meses }\end{array}$ & $\begin{array}{l}\text { TEV (3-12 meses previos) } \\
\text { Trombofilia no grave } \\
\text { (heterocigoto FV Leiden o } \\
20210 \text { protrombina) } \\
\text { TEV recurrente, TEV + } \\
\text { cáncer activo }\end{array}$ \\
\hline Bajo & Posición aórtica sin FR & $\begin{array}{l}\mathrm{CHA}_{2} \mathrm{DS}_{2} \text {-VASc } 1-4 \\
\text { Sin ACV/AIT previo }\end{array}$ & TEV >12 meses \\
\hline
\end{tabular}

ACV: ataque cerebrovascular; AIT: ataque isquémico transitorio; DM: diabetes mellitus; FA fibrilación auricular; FR: factor(es) de riesgo; IC: insuficiencia cardíaca; TEV: tromboembolia venosa; SAF: síndrome antifosfolipídico; FV Leiden: mutación G1691A del Factor V; 20210 protrombina: mutación G20210A de la protrombina.

dos los tubos de drenaje, comenzando conjuntamente con warfarina, dado que su efecto tiene latencia, y depende no sólo de las dosis utilizadas sino también de la respuesta del paciente al fármaco.

En pacientes de muy alto riesgo trombótico (que asocie 2 o más elementos de alto riesgo), puede iniciarse precozmente con heparina no fraccionada en bomba de infusión continua (BIC), antes de las 12 horas del posoperatorio, con estricta evaluación del riesgo hemorrágico y del KPTT cada 6 horas, intentando mantenerlo en 1,5-2 veces el valor normal.

Es obligatorio, antes de comenzar el tratamiento, conocer el recuento plaquetario, que preferiblemente debe ser mayor de 100.000 plaquetas $/ \mathrm{mm}^{3}$ en cuyo caso puede utilizarse dosis plena. Entre 50.000 y 100.000 plaquetas $/ \mathrm{mm}^{3}$ se comienza con precaución, y por debajo de 50.000 se debe esperar y controlar el número de plaquetas cada 24 horas.

De la misma manera, importa conocer los niveles de hemoglobina, vigilar las heridas quirúrgicas, descartando la presencia de sangrado manifiesto a través de los drenajes o retenido en pericardio y recurriendo a estudios de imagen ante la duda; pesquisar un derrame pleural o un sangrado digestivo.

La plaquetopenia producida por la CEC ocurre en el $22 \%-52 \%$ de los casos $^{(3)}$. Debe diferenciarse de la HIT que ocurre en 1\%-3\% de los casos ${ }^{(3,6,7)}$. Su correcta identificación es importante porque en la HIT, la mitad de los pacientes desarrollará complicaciones tromboembólicas.
El diagnóstico clínico de HIT debe plantearse frente a un descenso de más de $50 \%$ del recuento plaquetario en los siguientes 5-10 días de iniciada la heparinoterapia. Hay 2 formas de HIT: tipo I, con reducción transitoria y en general no significativa del número de plaquetas, y tipo II, más severa y frecuentemente acompañada de trombosis. En una revisión de 14.000 pacientes realizada por Sun y colaboradores, 15\% tenía anticuerpos (Ac) antiplaquetarios y sólo 4\% desarrolló trombosis. El hallazgo de Ac junto a plaquetopenia y trombosis se asoció a mayor mortalidad a los 30 días, comparado con la ausencia de Ac y plaquetopenia. El número de plaquetas se recupera a la semana de la suspensión de la heparina en el $90 \%$ de los $\operatorname{casos}^{(7,8)}$.

La eliminación del Ac anti-factor plaquetario 4 (FP4) de la sangre se produce en 2-3 meses.

El mecanismo se inicia con la formación de un complejo heparina-FP4 que genera una respuesta inmune mediante Ac inmunoglobulina $G$, con formación de un complejo heparina-FP4-Ac. Esto determina la activación de las plaquetas y liberación de trombina, generando un estado procoagulante. Su tratamiento será la suspensión de la heparinoterapia y su rotación a fondaparinux ${ }^{(7)}$.

\section{Uso de warfarina en el perioperatorio}

En el preoperatorio, si la warfarina (anticoagulante antivitamina K [AVK]) está en rango terapéutico, debe suspenderse 5 días antes del procedimiento. El uso 
de HBPM como puente comienza 24-48 horas después de la suspensión del AVK, ya que su administración inmediata provocaría un estado de anticoagulación excesiva. Lo más correcto será controlar el INR a las 48 horas de la suspensión e iniciar HBPM una vez que el INR esté por debajo de 2, y suspenderla 24 horas antes de la cirugía. Siempre es recomendable realizar un INR de control en las 24 horas previas a la cirugía, la cual puede realizarse con adecuada seguridad cuando el INR es $\leq 1,5$. En caso de tratarse de un paciente de muy alto riesgo trombótico, se puede utilizar como puente heparina no fraccionada en BIC, pudiendo suspenderse 6-8 horas antes de la cirugía y retomarla 12-24 horas después de la misma, si fuera necesario $^{(9,10)}$.

La warfarina debe reiniciarse en las primeras 24-48 horas del POCC, con frecuencia superpuesta a heparina. La dosis de inicio será de $5 \mathrm{mg} /$ día, que se reducirá a 2,5 mg en pacientes mayores de 75 años, peso menor de $50 \mathrm{~kg}$, disfunción hepática, INR inicial $\geq 1,5$ o necesidad de uso de antiplaquetarios asociados, antimicóticos u otra medicación que potencie su efecto.

Es importante tener presente que la vida media de la warfarina es de 35-80 horas, y la duración del efecto se puede prolongar durante 4-5 días ${ }^{(9)}$ (figura 1).

Los controles de INR durante la internación en cuidados intensivos o moderados se realizarán cada 24 horas, y se ajustará la dosis de acuerdo a los valores de INR; en general se aumenta cada 48 horas un escalón en la dosis a administrar. Logrado el rango terapéutico deseado, se suspenderá la heparinoterapia y en 5-7 días se realizará el siguiente control de INR. En caso de mantener el rango terapéutico deseado, se espaciarán los controles de INR, primero en 15 días y luego mensualmente.

También corresponde conocer los efectos de los anticoagulantes directos, que vienen ganando espacio en la anticoagulación del paciente cardiológico, especialmente en la FA no valvular (tabla 2).

Un aspecto destacable es la tasa de eliminación renal; su descenso hará obligado el ajuste de dosis, siendo esto más importante para los fármacos que tienen mayor eliminación renal, como el dabigatran. De la misma manera, el filtrado glomerular será muy importante a la hora de discontinuar la medicación en caso de cirugía de coordinación, por lo que el dabigatran requerirá mayores tiempos en caso de cirugía con alto riesgo de sangrado o con filtrado glomerular disminuido.

\section{Manejo de las complicaciones hemorrágicas en pacientes con anticoagulantes antivitamina $\mathrm{K}$}

Se requiere mucha atención para detectar y tratar la anticoagulación excesiva, con el agravante de que

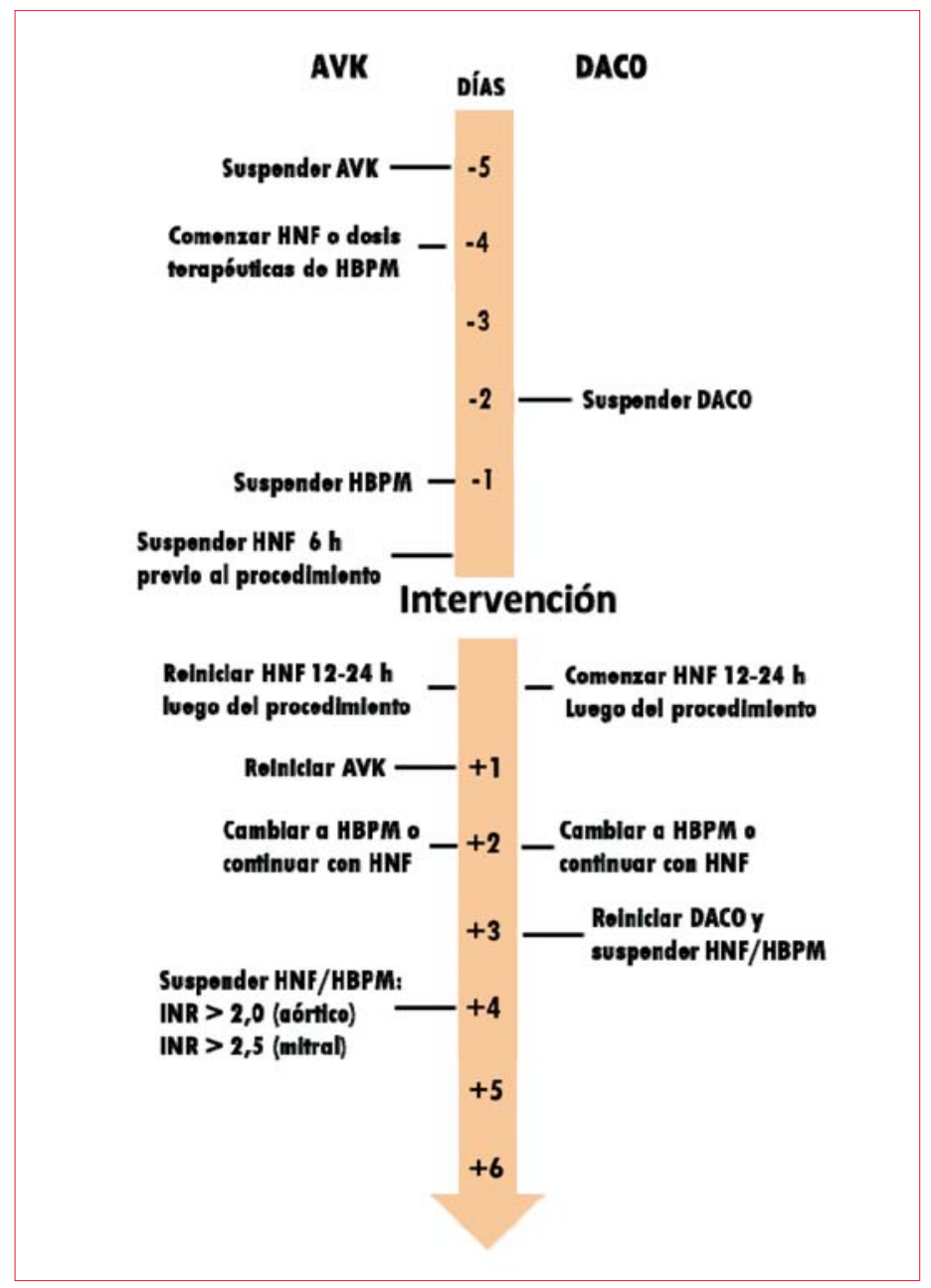

Figura 1. Esquema de manejo de la anticoagulación en el perioperatorio de cirugía cardíaca. Modificado de ${ }^{(9)}$. AVK: antivitamina K; DACO: anticoagulantes directos; HNF: heparina no fraccionada; HBPM: heparina de bajo peso molecular; INR: International Normalized Ratio.

se trata de un paciente en POCC con múltiples fuentes potenciales de sangrado. Se sugiere el siguiente esquema:

- INR < 5 sin sangrado: la conducta será la suspensión de AVK por 24-48 horas, y una vez logrado el rango deseado, reiniciar con dosis más bajas. Los controles del INR se realizan cada 24 horas hasta lograr la estabilidad en rango terapéutico, momento a partir del cual se pueden espaciar los controles.

- INR entre 5-9 sin sangrado: suspender AVK más de 48 horas; siempre es importante tener presente el motivo de la anticoagulación, ya que el uso indiscriminado de vitamina $\mathrm{K}$ nos expone a generar resistencia a la warfarina.

- Si el riesgo trombótico no es alto, se pueden utilizar bajas dosis de vitamina $\mathrm{K}$ vía oral (v/o) (2,5 mg) y realizar INR de control en 12 horas, plazo en el que se corrige el INR. 


\begin{tabular}{|c|c|c|c|c|}
\hline & Apixaban & Dabigatran & Edoxaban & Rivaroxaban \\
\hline Objetivo & Factor X & Trombina & Factor X & Factor X \\
\hline Biodisponibilidad & $51 \%-85 \%$ & $6 \%-8 \%$ & $60 \%$ & $80 \%$ \\
\hline T máx. (horas) & 3 & 2 & $1-3$ & $2-4$ \\
\hline Vida media (horas) & $9-14$ & $14-17$ & $5-11$ & $9-13$ \\
\hline Administración & 2 veces/día & 2 veces/día & Una vez/día & 1 o 2 veces/día \\
\hline Eliminación renal & $25 \%$ & $80 \%$ & $36 \%-45 \%$ & $33 \%$ \\
\hline Antídoto & Andexanet alfa & Idarucizumab & Andexanet alfa & Andexanet alfa \\
\hline $\begin{array}{l}\text { Discontinuación antes de } \\
\text { cirugía de coordinación (horas) }\end{array}$ & $\mathrm{Al}$ menos 48 & $\mathrm{Al}$ menos 48-96 & $\mathrm{Al}$ menos 48 & $\mathrm{Al}$ menos 48 \\
\hline
\end{tabular}

- INR $>8$ sin sangrado, se recomienda internación, ya que no es prudente el manejo ambulatorio por el eventual riesgo de sangrado que pueda poner en riesgo la vida del paciente.

- INR > 9 sin sangrado: el comportamiento es similar al anterior, pero se indica vitamina $\mathrm{K}$ 2,5-5 mg v/o.

- INR > 9 con sangrado: ingresar al paciente, suspender AVK 2-3 días, indicar vitamina $\mathrm{K}$ intravenosa (i/v), $10 \mathrm{mg}$ en $100 \mathrm{ml}$ de suero fisiológico en 15 minutos. Según la gravedad del sangrado, se puede utilizar plasma fresco (precaución: pueden requerirse varios volúmenes para corregir el INR) o el uso de complejo protrombínico, que corrige rápidamente el INR, pero expone al riesgo de trombosis. En estos casos es recomendable siempre interconsulta con hemoterapia ${ }^{(11,12)}$ (figura 2 ).

- INR en rango con sangrado: en este caso también se requiere la suspensión del AVK por el menor tiempo posible, acorde al motivo de anticoagulación. Siempre debe buscarse la causa local del sangrado, ya que la mucosa sana no sangra con un INR en rango y especialmente estos pacientes no deben recibir vitamina $\mathrm{K}$.

Se define el sangrado mayor según la clasificación de la Sociedad Internacional de Hemostasis y Trombosis, como descenso de la hemoglobina $>2 \mathrm{~g} / \mathrm{dl}$, bajo gasto o hipotensión, sangrado en logia no compresible, pericardio, retroperitoneo o intracraneano. Estos pacientes requieren reposición de concentrados de glóbulos rojos, vitamina $\mathrm{K}$ i/v o complejo protrombínico acorde al rango de INR y solución quirúrgica de la causa del sangrado si lo requiere ${ }^{(12)}$.

La vitamina $\mathrm{K}$ i/v solo se utilizará en casos de emergencia vital, sangrado activo con INR elevado,
INR > de 20 aun sin sangrado o corrección del INR previo a una cirugía de urgencia.

\section{Tratamiento anticoagulante en escenarios específicos}

\section{Prótesis valvulares}

Como se ve en la tabla 3 , los pacientes con prótesis mecánica aórtica o mitral con factores de riesgo trombótico asociados, requieren aumento del rango terapéutico. Para válvula protésica aórtica se eleva el INR a valores medios de $3(2,5-3,5)$ y para la posición mitral se evalúa la asociación de ácido acetil salicílico (AAS) $100 \mathrm{mg}$. Sin duda, cada vez que utilicemos asociación con antiplaquetarios, aumentaremos el riesgo hemorrágico. Las prótesis mecánicas tienen indicación excluyente de warfarina. Los anticoagulantes directos no tienen lugar en este contexto $^{(4,11,13)}$.

Cuando nos referimos a las bioprótesis, la posición mitral parece tener una conducta clara y definida: requiere anticoagulación con warfarina por un plazo de 3-6 meses, comenzando conjuntamente HBPM en el posoperatorio, como ya fuera mencionado. Luego de ese período, el paciente quedará antiagregado con AAS $100 \mathrm{mg} /$ día, indicación IIa B-NR de la American Heart Association/American College of Cardiology (AHA-ACC) ${ }^{(4)}$.

La misma conducta se aplica a los pacientes en los que se realizan plastias mitrales, con rangos de INR 2-3.

Aún es discutida la conducta en la bioprótesis aórtica. Tanto la AHA como las Guías Canadienses de Cardiología están a favor del uso de anticoagula- 


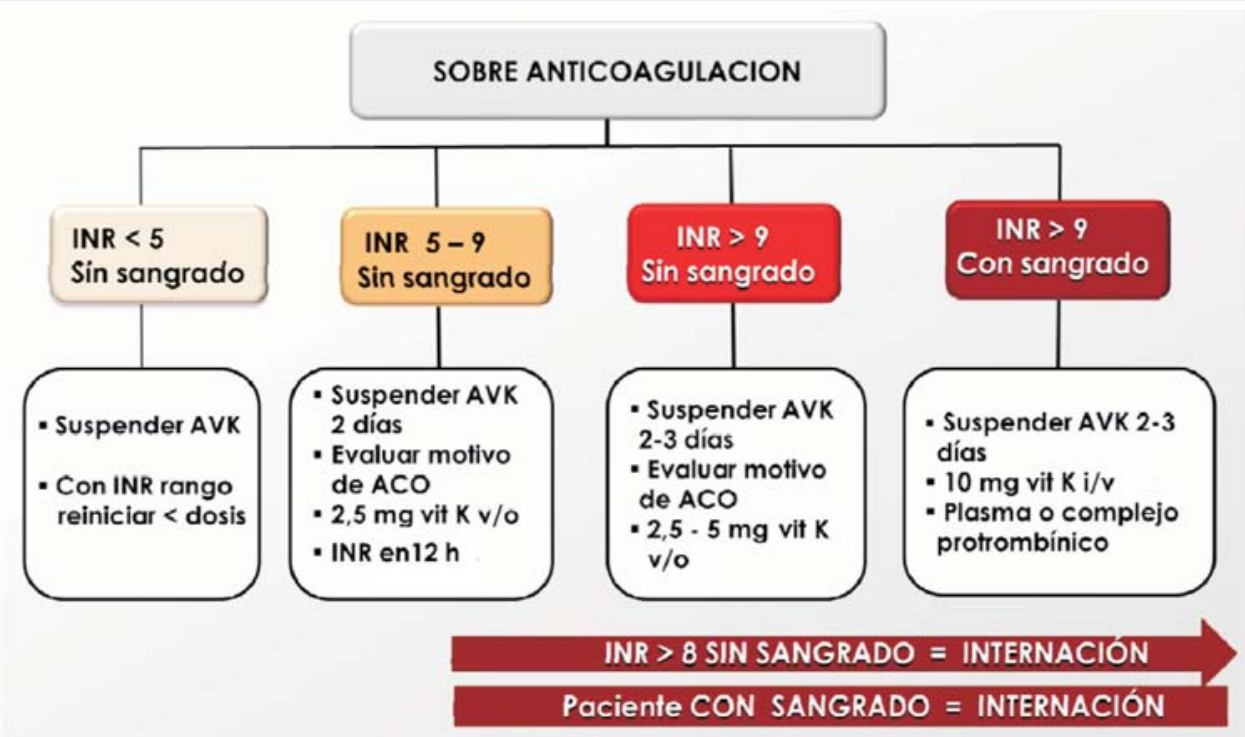

Figura 2. Algoritmo de manejo del paciente con INR supraterapéutico.

Tabla 3. Valores objetivo de INR según tipo y posición de la prótesis implantada.

\begin{tabular}{lccccc}
\hline & \multicolumn{2}{c}{ Posición aórtica } & \multicolumn{2}{c}{ Posición mitral } & Mitroaórtica \\
\cline { 2 - 5 } & Ritmo sinusal & Con factores de riesgo & Ritmo sinusal & Con factores de riesgo \\
\hline & & & \multicolumn{2}{c}{ Asociar AAS $100 \mathrm{mg}$} \\
Prótesis mecánica & $2-3$ & $2,5-3,5$ & $2,5-3,5$ & $2,5-3,5$ & $2,5-3,5$ \\
Prótesis biológica & $2-3$ & $2-3$ & $2-3$ & $2,5-3,5$ & $2,5-3,5$ \\
Plastia mitral & & & $2-3$ & $2-3$ &
\end{tabular}

ción en este contexto por un período de 3-6 meses (IIa B) (4).

Sin embargo, la Sociedad Europea de Cardiología y las guías de tratamiento antitrombótico de la American College of Chest Physicians de 2012 apoyan el uso sistemático de AAS $100 \mathrm{mg} /$ día, indicación IIa C ${ }^{(11)}$.

Un estudio de Cleveland Clinic que incluyó 31.740 pacientes con SVA por bioprótesis tratados con AVK vs AAS por un plazo de 3 meses, observó una incidencia de trombosis de $1 \%$ vs $1,5 \%$ ( $\mathrm{p}=$ $0,85)$ y mortalidad de $3,5 \%$ vs $2,9 \%(\mathrm{p}=0,14)$ respectivamente.

El sangrado fue de $2,5 \%$ vs $1,1 \%$ ( $p<0,0001$, favoreciendo al AAS). Concluyen los autores que no se demostró beneficio con AVK en esta población ${ }^{(14)}$.

En 943 pacientes del registro Partner 2, los pacientes dados de alta sin AVK tuvieron gradientes transvalvulares $10 \mathrm{mmHg}$ más altos al mes y al año de la cirugía ${ }^{(15)}$. Este resultado no pudo ser reproducido en un estudio prospectivo realizado en nuestro medio en pacientes con SVA quirúrgica por bioprótesis y presentado recientemente en el $35^{\circ}$ Congreso Uruguayo de Cardiología(16).

Actualmente parece razonable que un paciente con bioprótesis aórtica y bajo riesgo de sangrado, reciba AVK por un lapso de tres meses, y un paciente de mayor riesgo sea tratado con AAS.

Cuando se opta por AVK, dado que la bioprótesis aórtica tiene menor riesgo trombótico, se sugiere iniciar warfarina, a partir del posoperatorio, sin asociar HBPM.

\section{Fibrilación auricular posoperatoria}

La FA en el POCC es frecuente (20\%-50\% de los casos). Su presencia prolonga la estadía hospitalaria, aumenta la morbimortalidad y es marcador de FA a largo plazo después de la cirugía (17).

La FA hemodinámicamente inestable no admite otra conducta que la cardioversión eléctrica (CVE) inmediata. En los pacientes hemodinámicamente estables, el control de frecuencia o de ritmo se reali- 
za de igual modo que fuera del contexto quirúrgico. Para el control de frecuencia, los betabloqueantes y los anticálcicos tienen preferencia sobre la digoxina. Para la reversión farmacológica a ritmo sinusal, la amiodarona es la droga ideal en este escenario. En los pacientes con FA de menos de 24-48 horas de instalada y hemodinámicamente estable, se realizará una carga de amiodarona $\mathrm{i} / \mathrm{v}$ en dosis de 900 a $1.200 \mathrm{mg}$ en las primeras 24 horas. Si se logra reversión a ritmo sinusal, se sugiere mantener el tratamiento por un lapso aproximado de 60 días, en general a una dosis de $200 \mathrm{mg} /$ día.

Se suspenderá antes de ese tiempo en caso de efectos secundarios que incluyen: disfunción tiroidea, intervalo QT >480 ms y bradicardia(17).

De no lograr la reversión a ritmo sinusal con amiodarona, es preferible la CVE antes del requerimiento obligado de anticoagulación. Pasadas las 48 horas, el paciente necesariamente debe recibir anticoagulación con HBPM asociada a AVK hasta lograr el rango de INR entre $2-3$. De igual modo, puede recibir anticoagulantes directos (DACO) en la FA no valvular, en las dosis que habitualmente utilizamos fuera de la cirugía cardíaca.

El paciente que instala FA aguda en la UCI durante la etapa de asistencia respiratoria mecánica, se beneficia de la CVE precoz, aprovechando la sedación de las primeras horas del posoperatorio y siempre asociando una carga de amiodarona i/v.

Tienen indicación de anticoagulación los pacientes portadores de FA presente desde el preoperatorio, la FA aguda con más de 24 horas de instalada hasta definir la oportunidad de CVE y siempre si tiene más de 48 horas, la FA paroxística con 2 o más episodios y score $\mathrm{CHA}_{2} \mathrm{DS}_{2}$-VASc $>3$. Estos pacientes requieren el inicio conjunto de HBPM y warfarina, hasta lograr el rango entre 2-3 de la misma manera que se definió previamente.

Si se cuenta con DACO, se pueden utilizar en la FA no valvular del posoperatorio . La FA que ocurre en el contexto de bioprótesis aórtica también podría beneficiarse de DACO ${ }^{(18,19)}$, aunque se espera evidencia más concluyente al respecto. Para pacientes con bioprótesis mitral, la recomendación está aún a favor de la warfarina por los primeros 3 meses. Luego de este período, se puede hacer el cambio a DACO. El beneficio de estos fármacos es la anticoagulación estable y el no requerir controles de INR, como ha sido demostrado en sus trabajos originales.

\section{Prevención de la enfermedad tromboembólica venosa}

Existen diferentes reglas de predicción para la prevención de la enfermedad tromboembólica venosa (ETEV) del posoperatorio. Todas ellas son de utili- dad y nos permiten reconocer la población de mayor riesgo de desarrollar trombosis venosa profunda (TVP) y/o embolia pulmonar (TEP). El score de Caprini es un buen sistema de puntuación que estratifica el riesgo de ETEV en los pacientes quirúrgicos ${ }^{(20)}$.

Una revisión que compara la prevalencia de TVP en diferentes escenarios posoperatorios, analiza un total de 2.669.772 pacientes, de los cuales 18.670 desarrollaron TVP (0,69\%). Cuando se compara cirugía general versus cirugía vascular y cirugía cardíaca, se comprueba que la prevalencia de TVP fue $0,66 \%, 0,99 \%$ y $2 \%$ respectivamente, con $\mathrm{OR}=3$ para la cirugía cardíaca y $\mathrm{p}<0,001$ de significación estadística ${ }^{(21)}$.

Los autores concluyen en la necesidad de realizar tromboprofilaxis en el POCC.

La mayor parte de las TVP pasan inadvertidas y sólo $20 \%$ son clínicamente evidentes, pero todas están en riesgo de desarrollar TEP, lo que condiciona mayor morbimortalidad y la posibilidad de complicaciones futuras, como hipertensión pulmonar y síndrome postrombótico.

Este estudio destaca factores de riesgo potentes para el desarrollo de ETEV: historia de enfermedad arterial periférica de miembros inferiores, ACV previo, paraplejia, tratamiento quimioterapéutico actual, sepsis perioperatoria, cirugía de emergencia, tiempos quirúrgicos prolongados y estadía prolongada en cama, coincidentes con varios de los factores de riesgo que describe Caprini en su score ${ }^{(19)}$. Consideramos muy útil este score para promover el uso de tromboprofilaxis en el POCC. Todo paciente que no deambula a las 48 horas, debe recibir HBPM en dosis de tromboprofilaxis: $40 \mathrm{mg}$ s/c día para $<70 \mathrm{~kg}$ de peso y $60 \mathrm{mg}$ $\mathrm{s} / \mathrm{c}$ día para mayor peso. No hay dosis establecidas para obesos de más de $100 \mathrm{~kg}$, aunque probablemente requieran dosis mayores. La dosificación referida debe reducirse a la mitad en pacientes con clearence renal menor de $30 \mathrm{ml} / \mathrm{min} / 1,73 \mathrm{~m}^{2}$.

\section{Conclusiones}

La anticoagulación en el POCC representa todo un reto y requiere un adecuado balance entre riesgo y beneficio. Cada paciente debe recibir su "traje a medida", y a pesar de ello no estamos exentos de posibles complicaciones hemorrágicas. El trabajo en equipo, multi e interdisciplinario y la protocolización de las medidas a tomar, facilitan el manejo y reducen las complicaciones, muchas de ellas evitables. 
Pablo Asadurian Tchakirian,

https://orcid.org/ 0000-0002-0959-0890

Este artículo fue aceptado para su publicación por: Editor asociado Dr. Jorge Estigarribia

\section{Bibliografía}

1. Baumann K, Karkouti K, Tweddell J, Massicotte M. Antithrombotic therapy management of adult and pediatric cardiac surgery patients. J Thromb Haemost. 2018; 16: 2133-46. doi: 10.1111/jth.14276

2. Barreto-Filho JA, Wang Y, Dodson JA, Desai MM, Sugeng L, Geirsson A, et al. Trends in aortic valve replacement for elderly patients in the United States, 1999-2011. JAMA 2013; 310: 2078-85. doi: 10.1001/jama.2013.282437

3. Ávila ML, Shah V, Brandao LR. Systematic review on heparin induced thrombocytopenia in children: a call to action. J Thromb Haemost. 2013; 11: 660-9. doi:10.1111/jth.12153

4. Nishimura RA, Otto CM, Bonow RO, Carabello BA, Erwin J P III, Fleisher LA, et al. 2017 AHA/ACC Focused Update of the 2014 AHA/ACC Guideline for the Management of Patients With Valvular Heart Disease. A Report of the American College of Cardiology/American Heart Association Task Force on Clinical Practice Guidelines. Circulation. 2017; 135: e1159-e1195. doi: 10.1161/ CIR.0000000000000503

5. Vivas D, Roldán I, Ferrandis R, Marýne F, Roldán V, Tello-Montoliue A, et al. Manejo perioperatorio y periprocedimiento del tratamiento antitrombótico: documento de consenso de SEC, SEDAR, SEACV, SECTCV, AEC, SECPRE, SEPD, SEGO, SEHH, SETH, SEMERGEN, SEMFYC, SEMG, SEMICYUC, SEMI, SEMES, SEPAR, SENEC, SEO, SEPA, SERVEI, SECOT y AEU. Rev Esp Cardiol. 2018; 71 (7): 553-64. doi: 10.1016/j.recesp.2018. 01.001

6. Ivascu NS, Fitzgerald M, Ghadimi K, Prakash P, Evans A, Goeddel L, et al. Heparin-Induced Thrombocytopenia: A Review for Cardiac Anesthesiologists and Intensivists. J of Cardiothorac Vasc Anesth. 2019; 33: 511-20. doi: 10.1053/ j.jvca.2018. 10. 035

7. Seigerman M, Cavallaro P, Itagaki S, Chung I, Chikwe J. Incidence and outcomes of heparin-induced thrombocytopenia in patients undergoing cardiac surgery in North America: An analysis of the nationwide inpatient sample. J Cardiothorac Vasc Anesth. 2014; 28: 98-102. doi: 10.1053/j.jvca.2013. 07.021

8. Sun X, Hill PC, Taylor-Pane KS, Corso P, Lindsay J. Heparin-induced thrombocytopenia in con- temporary cardiac surgical practice and experience with a protocol for early identification. Am J Cardiol. 2016; 117: 305-9. doi: 10.1016/j.amjcard.2015.10.047

9. Sousa-Uva M, Head SJ, Milojevic M, Collet JP, Landoni G, Castella M, et al. 2017 EACTS Guidelines on perioperative medication in adult cardiac surgery. European Journal of Cardio-Thoracic Surgery 2017; 00:1-29. doi:10.1093/ejcts/ezx314

10. Douketis JD, Spyropoulos AC, Spencer FA, Mayr M, Jaffer AK, Eckman MH, et al. Perioperative Management of Antithrombotic Therapy: Antithrombotic Therapy and Prevention of Thrombosis, 9th ed.: American College of Chest Physicians Evidence-Based Clinical Practice Guidelines. Chest 2012; 141: e326S-e350S. doi:10.1378/chest.11-2298

11. Baumgartner H, Falk V, Bax J, De Bonis M, Hamm CH, Holm PJ, et al. 2017 ESC/EACTS Guidelines for the management of valvular heart disease. The Task Force for the Management of Valvular Heart Disease of the European Society of Cardiology (ESC) and the European Association for Cardio-Thoracic Surgery (EACTS). European Heart Journal 2017; 38: 2739-91. doi:10.1093/eurheartj/ehx391

12. Holbrook A, Schulman S, Witt DM, Vandvik PO, Fish J, Kovacs MJ, et al. Evidence-Based Management of Anticoagulant Therapy : Antithrombotic Therapy and Prevention of Thrombosis, $9^{\text {th }}$ ed: American College of Chest Physicians Evidence-Based Clinical Practice Guidelines. Chest 2012; 141: e152S-e184S. doi:10.1378/chest.11-2295

13. Saksena D, Muralidharan S, Mishra YK, Kanhere V, Mohanty B, Srivastava CP, et al. Anticoagulation Management in Patients with Valve Replacement. J Assoc Phys India 2018; 66: 59-74

14. Gryaznov A, Saeyeldin A, Abdelbaky M, Zafar M, Tanweer M, Imrana M, et al. Antithrombotic Therapy after Bioprosthetic Aortic Valve Replacement: A Therapeutic Morass. Cardiology 2018; 140: 213-21. doi:10.1159/000490924

15. Chakravarty T, Patel A, Kapadia S, Raschpichler M, Smalling R, Szeto W, et al. Anticoagulation After Surgical or Transcatheter Bioprosthetic Aortic Valve Replacement. JACC 2019; 74 (9): 1190-200. doi: 10.1016/j.jacc.2019.06.058

16. Fernández A, Loza G, Arocena MJ, Canessa M, Gómez A, Soca G, et al. Anticoagulación en bioprótesis valvular aórtica. Ensayo clinico randomizado multicéntrico. Presentado en el $35^{\circ}$ Congreso Uruguayo de Cardiología, № 161. Nov. 9, 2019. Punta del Este, Uruguay.

17. Gillinov AM, Bagiella E, Moskowitz AJ, Raiten JM, Groh MA, Bowdish ME, et al. Rate Control versus Rhythm Control for Atrial Fibrillation after Cardiac Surgery. N Engl J Med. 2016; 374 (20): 1911-21. doi:10.1056/NEJMoa1602002 
18. Guimarães PO, Pokorney SD, Lopes RD, Wojdyla DM, Gersh BJ, Giczewska A, et al. Efficacy and safety of apixaban vs warfarin in patients with atrial fibrillation an prior bioprosthetic valve replacement or valve repair: Insights from ARISTOTLE trial. Clinical Cardiology 2019; 42: 568-71. doi:10.1002/clc.23178

19. Russo V, Carbone A, Attena E, Rago A, Mazzone C, Proietti R, et al. Clinical benefit of direct oral anticoagulants versus vitamin $\mathrm{K}$ antagonists in patients with atrial fibrillation and bioprosthetic heart valves. Clin Therap. 2019; 41 (12): 2549-57.

doi: 10.1016/j.clinthera.2019.10.008

20. Caprini J A. Venous Thromboembolism. Thrombosis Risk Assessment as a Guide to Quality Patient Care. Diseases Month February/March 2005. doi: j.disamonth.2005.02.002

21. Aziz F, Patel M, Ortenzi G, Reed A. Incidence of Postoperative Deep Venous Thrombosis Is Higher among Cardiac and Vascular Surgery Patients as Compared with General Surgery Patients. Ann Vasc Surg. 2015; 29: 661-9. doi: 10.1016/ j. avsg.2014.11.025 